\title{
Platelet functional alterations in a Bernard-Soulier syndrome patient with filamin A mutation
}

\author{
Jiaming Li, KeSheng Dai, Zhaoyue Wang ${ }^{*}$, Lijuan Cao, Xia Bai and Changgeng Ruan
}

\begin{abstract}
Defects in filamin A (FLNA) gene could lead to low platelet counts and decreased surface expression of glycoprotein (GP) Iba. Here, we report and investigate the FLNA genomic alteration of a case with Bernard-Soulier syndrome (BSS), a rare hereditary bleeding disorder caused by quantitative or qualitative abnormalities in the GP Ib-IX-V receptor. DNA sequencing analysis reveals the presence of a GP Iba c.987G > A mutation and a FLNA c.1582 $\mathrm{G}>$ A mutation in this patient. Transient transfection studies show that GP Iba c.987G > A mutation abolishes the surface expression of GP Iba on the transfected CHO cells. On the other hand, abnormal responses to collagen, including the platelet aggregation, secretion, and GP VI signaling pathways, are associated with FLNA c.1582G > A mutation. Our findings confirm a central role for FLNA in platelet-adhesive functions. The interaction between FLNA and GP Iba in platelets deserves to be investigated.
\end{abstract}

Keywords: Bernard-Soulier syndrome, Platelets, Glycoprotein Ib-IX complex, Thrombocytopenia, Mutation

\section{To the Editor}

Filamin A (FLNA), a dimeric actin crosslinking protein, anchors the platelet adhesion glycoprotein (GP)Ib-IX-V receptor to actin cytoskeleton. The GP Ibo-FLNA interaction is essential for the platelet adhesion to von Willebrand factor that binds the GP Ib-IX-V receptor, for normal signal transduction reactions involved in platelet activation, and for maintaining normal platelet shape and integrity [1]. The absence of or mutations in the GP Ib-IX-V receptor or FLNA gene is responsible for Bernard-Soulier syndrome (BSS) or macrothrombocytopenia [2], respectively. We described here a female BSS case with FLNA mutation, showing her platelet functional defects in the platelet aggregation, secretion, and GP VI signaling pathways.

Patient's platelet counts ranged between 20 and $30 \times$ $10^{9} / \mathrm{L}$, and enlarged platelets were observed in her blood film. Platelet aggregation on ristocetin was null. Collagen was decreased to induce platelet aggregation $(0.5 \mu \mathrm{g} / \mathrm{ml}$, $17 \% ; 1 \mu \mathrm{g} / \mathrm{ml}, 37 \%$ versus $45 \%, 70 \%$ of controls). The GPVI-specific agonist, convulxin, was also decreased

\footnotetext{
* Correspondence: zwang201411@126.com

Jiangsu Institute of Hematology, The First Affiliated Hospital of Soochow University, Key Laboratory of Thrombosis and Hemostasis of Ministry of Health, 188 Shizi Street, Suzhou 215006, China
}

(400 PM, 10 \%; 800 PM, 40 \% versus $55 \%$, 70 \% of controls). Platelets released 33-86 \% less ATP than control platelets (Fig. 1a). The GP Ibo expression was practically undetectable on the platelet surface. DNA sequencing analysis revealed the presence of a new GP Ib $\alpha$ c.987G > A mutation in a homozygous state and a FLNA c.1582 $\mathrm{G}>\mathrm{A}$ mutation in a heterozygous state (Fig. 1b).

To establish that GP Ib $\alpha$ c.987G > A mutation was responsible for the lack of GP Iba surface expression in the patient's platelets, we expressed in Chinese hamster ovary $(\mathrm{CHO})$ cells the mutant or normal GP Ib $\alpha$ cDNA together with normal human cDNAs of GP $\mathrm{Ib} \beta$ and GPIX. Only trace amounts of GP Ib $\alpha$ and GPIX was detected on $\mathrm{CHO}$ cells harboring the mutation, but truncated GP Ib $\alpha$ and normal GPIX was present in the $\mathrm{CHO}$ cell lysates (Fig. 1c). Together, these data could suggest shorten GP Ib $\alpha$ and GPIX were synthesized, but failed to be anchored and inserted into the plasma membrane.

A question raised by our observation was that patient's platelet responses to collagen were impaired, despite a normal amount of platelet GPVI and FcR $\gamma$ chain in her platelets. Several lines of evidence demonstrated that FLNA acted as a signaling scaffold for GPVI through interaction with tyrosine kinase Syk [3]. In our study, 


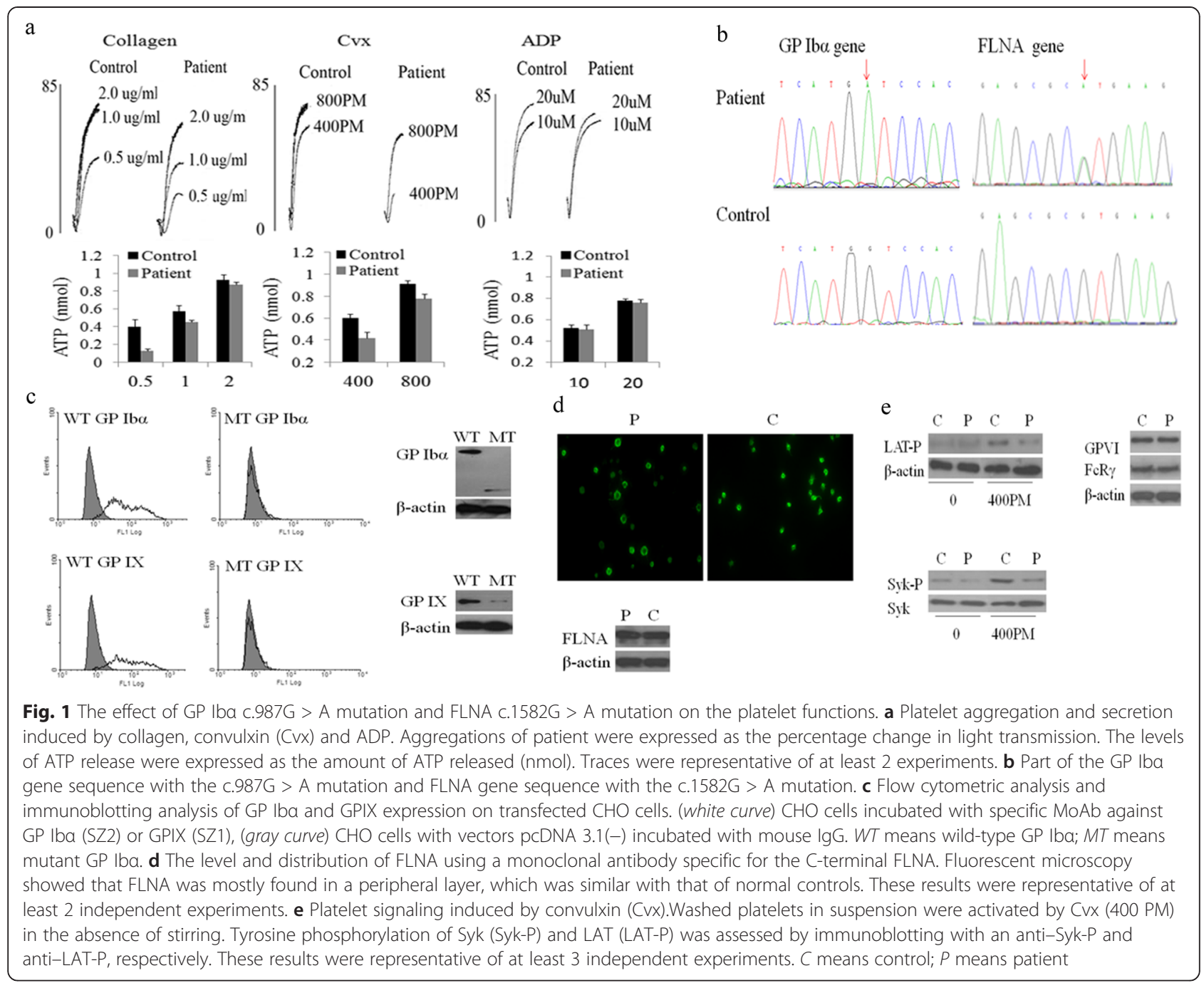

the normal level and regular distribution of FLNA was detected in the patient's platelets (Fig. 1d). However, the phosphorylation of Syk was low ( $41 \%$ of control) in the platelet signaling pathway of GPVI induced by Cvx (400 $\mathrm{pmol} / \mathrm{L})$. Similarly, the phosphorylation of LAT, a direct substrate of Syk, was decreased ( $45 \%$ of control) (Fig. 1e). FLNA could bind to the tyrosine kinase Syk through its immunoglobulin-like repeat $3-5$ in platelets [4]. In our patient, the identified FLNA c.1582G > A, an Ig repeat 3 mutation, interfered with the Ig repeats engaged in signaling of GPVI-collagen interaction. In turn, platelet aggregation and ATP secretion on collagen was interrupted. We thus conclude that abnormal responses to collagen were associated with FLNA c.1582G > A mutation.

To the best of our knowledge, this was the first report of a FLNA mutation causing abnormal response to collagen in a BSS patient. According to our results, we demonstrated that the synergistic effect of both GP Ib $\alpha$ c. $987 \mathrm{G}>$ A mutation and FLNA c.1582G > A mutation could lead to the platelet functional alteration, including aggregation, secretion, and the GPVI signaling pathway. The relationship between GP Ib $\alpha$ and FLNA should constitute an area of interest for future studies.

\section{Competing interests}

The authors declare that they have no competing interests.

\section{Authors' contributions}

$J$ performed research and collected clinical data. LC, XB, and CR participated in the research. KD analyzed the data. ZW designed the study and wrote the paper. All authors read and approved the final manuscript.

\section{Acknowledgements}

This work was funded by the Jiangsu Provincial Special Program of Medical Science (BL2012005), the Jiangsu Province's Key Medical Center (ZX201102), and the Priority Academic Program Development of Jiangsu Higher Education Institutions (PAPD).

Received: 4 June 2015 Accepted: 11 June 2015

Published online: 02 July 2015

\section{References}

1. Williamson D, Pikovski I, Cranmer SL, Mangin P, Mistry N, Domagala T, et al. Interaction between platelet glycoprotein Iba and filamin-1 is essential for 
glycoprotein Ib/IX receptor anchorage at high shear. J Biol Chem. 2002;277:2151-9.

2. Nurden P, Debili N, Coupry I, Bryckaert M, Youlyouz-Marfak I, Solé G, et al. Thrombocytopenia resulting from mutations in filamin A can be expressed as an isolated syndrome. Blood. 2011;1 18:5928-37.

3. Ichinohe T, Takayama H, Ezumi Y, Arai M, Yamamoto N, Takahashi H, et al. Collagen-stimulated activation of Syk but not c-Src is severely compromised in human platelets lacking membrane glycoprotein VI. J Biol Chem. 1997;272:63-8

4. Falet H, Pollitt AY, Begonja AJ, Weber SE, Duerschmied D, Wagner DD, et al. A novel interaction between FLNA and Syk regulates platelet ITAMmediated receptor signaling and function. J Exp Med. 2010;207:1967-79.

\section{Submit your next manuscript to BioMed Central and take full advantage of:}

- Convenient online submission

- Thorough peer review

- No space constraints or color figure charges

- Immediate publication on acceptance

- Inclusion in PubMed, CAS, Scopus and Google Scholar

- Research which is freely available for redistribution 